Proc. 15th Int. Conference on Defects Recognition, Imaging and Physics in Semiconductors, Warsaw, Poland 2013

\title{
Effect of Secondary Electroluminescence on Cathodoluminescence and Other Luminescence Measurements
}

\author{
M. Pluska, A. Czerwinski, A. Szerling, J. Ratajczak and J. Kątcki \\ Institute of Electron Technology, al. Lotników 32/46, 02-668 Warszawa, Poland
}

\begin{abstract}
Cathodoluminescence and photoluminescence measurements are commonly accepted as revealing local properties of a specimen region excited by a beam of electrons or photons. However, in the presence of a strong electric field (e.g. a junction) an electron (or light/laser) beam-induced current is generated, which spreads over the structure. A secondary non-local electroluminescence, generated by this current and detected together with the expected luminescence signal, may strongly distort measurement results. This was confirmed by cathodoluminescence measurements on test structures prepared by focused ion beam on AlGaAs/GaAs/InGaAs laser heterostructures. Methods for minimizing the distortion of measured luminescence signals are presented.
\end{abstract}

DOI: $10.12693 /$ APhysPolA.125.1027

PACS: 78.60.Hk, 85.30.De, 61.72.-y, 68.37.Hk

\section{Introduction}

Luminescence measurements belong to the most sensitive, non-destructive methods for analyzing the local properties of semiconductors, usually by sample scanning with focused beam of light or particles. Cathodoluminescence (CL) and photoluminescence (PL) are measured by a point-excitation with an electron or laser/light beam, respectively, followed by the detection of emitted photons.

It is commonly assumed that the registered luminescence originates from the close neighborhood of the excitation point [1], where the region size corresponds to the minority carrier diffusion length (in many cases of the order of nanometers). The reported high spatial resolutions of CL (10 nm for InGaN/GaN multiple quantum well structures) [2], and PL measurements $(30 \mathrm{~nm}$ for near-field PL of InAs/GaAs quantum dots) [3] seem to confirm this view. However, this paper shows that the phenomenon that can be called an emission of secondary electroluminescence (EL) may strongly affect CL (as well as PL) measurements. This effect extends the region of luminescence emission much wider or shifts it far away from the excitation point, even as much as hundreds of micrometers (with the size or magnitude of shift depending on the measured structure). This phenomenon impacts the measurements of all semiconductor structures or materials containing regions of strong electric field (heterojunctions, $p-n$ junctions, grain boundaries, etc.).

Because the effect occurs when the electron-hole $(\mathrm{e}-\mathrm{h})$ pairs are generated and separated in a strong electric field, it occurs regardless the excitation method used (i.e. for CL, PL, X-ray luminescence or ionoluminescence measurement techniques) and therefore may impact numerous measurements.

Besides confirming the occurrence of the phenomenon in CL measurements, this paper also presents methods for minimizing the distortion of measurement results, regardless the excitation method.
It has been reported that in the case of specimens with a built-in strong electric field the CL intensity may be affected by phenomena which occur even far from the excitation point [4]. The generation of electron beam induced current (EBIC) in such structures is competitive with the recombination of $\mathrm{e}-\mathrm{h}$ pairs, and therefore the EBIC outflow causes CL quenching.

In this paper we report that while the EBIC reduces the CL signal emitted from the region near the excitation point, EBIC spreading causes the electroluminescence to be emitted even far from the excitation point.

\section{Experimental}

In order to investigate EL emission during CL measurements, two test structures were prepared on epitaxial wafers of $\mathrm{AlGaAs} / \mathrm{GaAs}$ with $8 \mathrm{~nm}$ thick InGaAs quantum well $(\mathrm{QW})$, manufactured with molecular beam epitaxy (MBE). The wafers were at the intermediate stage of production of separate confinement heterostructure single quantum well (SCH-SQW) laser heterostructures. The first test structure consisted of mesa stripes (Fig. 1a) in a structure in which cladding regions and junctions at the region of lateral confinements were completely etched out (corresponding to cross-section denoted by $y$ in Fig. 1b). Most of each mesa was covered with metallization, but several uncovered narrow regions were left without the metallization (corresponding to cross-section denoted by $x$ in Fig. 1b). Bonding wires were attached to several metallized fields to enable also standard EL measurements. Thus, the test structure through electrical contacts at the mesa top and the structure bottom may be externally biased (to link results of such measurement with the phenomenon of secondary EL) or may be short-circuited by an external circuit.

The second test structure was prepared for a similar set of manufactured layers but with the upper cladding layer in the region outside the mesa being only partially removed by etching. Using the focused ion beam 


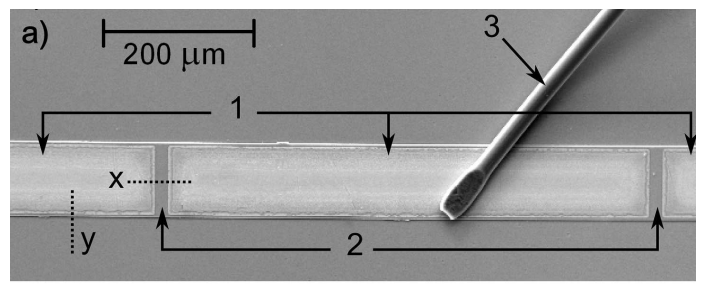

b)

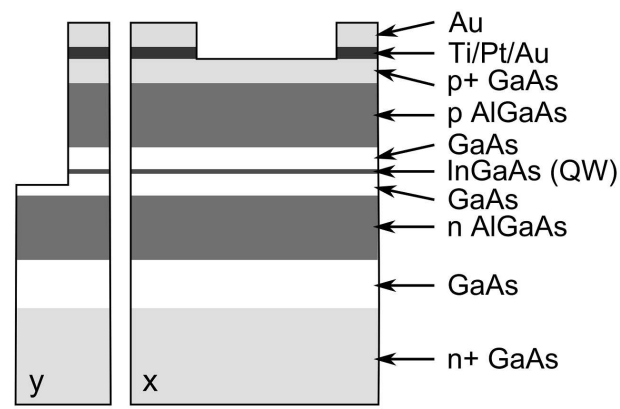

Fig. 1. (a) The SEM image of the first test structure (the inset shows drawing of its perspective projection). Specific regions of the structure are marked with numbers: 1 - mesa stripe with metal overlayer and contact, 2 - narrow regions of mesa uncovered by metallization, 3 - bonding wire providing electrical connection. (b) Cross-sections of the structure along the lines marked with the letters $x$ and $y$ in part (a).

(FIB) technique (with Helios NanoLab instrument), several cuts about $2 \mu \mathrm{m}$ deep were made to produce five stripes of $80 \mu \mathrm{m}$ length each (Fig. 2a). The upper layers in each stripe were separated from the other stripes and from the rest of the sample (Fig. 2b). The stripe widths were: $10,10,15,20$, and $25 \mu \mathrm{m}$. All were coated in the FIB chamber, first with a very thin (transparent to light) dielectric layer and then with an approximately $0.5 \mu \mathrm{m}$ thick platinum layer (opaque to light). One $10 \mu \mathrm{m}$ stripe was entirely coated with platinum, while each of the other four stripes at one end had an uncoated window of $10 \times 10 \mu \mathrm{m}^{2}$. The platinum coating was deposited to avoid detection of the local luminescence from the covered regions. Electrons with energy $30 \mathrm{keV}$ come across the $0.5 \mu \mathrm{m}$ thick Pt layer and create electron-hole pairs in the semiconductor, but the transmittance of this Pt layer is negligible for photons emitted from the semiconductor in the wavelength range $700-980 \mathrm{~nm}$. The dielectric underlayer protected the stripes against short circuiting by Pt layer.

The luminescence signal (due to CL and EL phenomena) for used test structures was detected using an Oxford Instruments MonoCL2 system in Philips XL30 SEM.

\section{Method}

Typically, the luminescence detector collects signals emitted from a specimen area of several hundred $\mu \mathrm{m}$ in size. Therefore, the detected luminescence consists of the intended (e.g. CL or PL) signal, together with the secondary (i.e. EL) components.

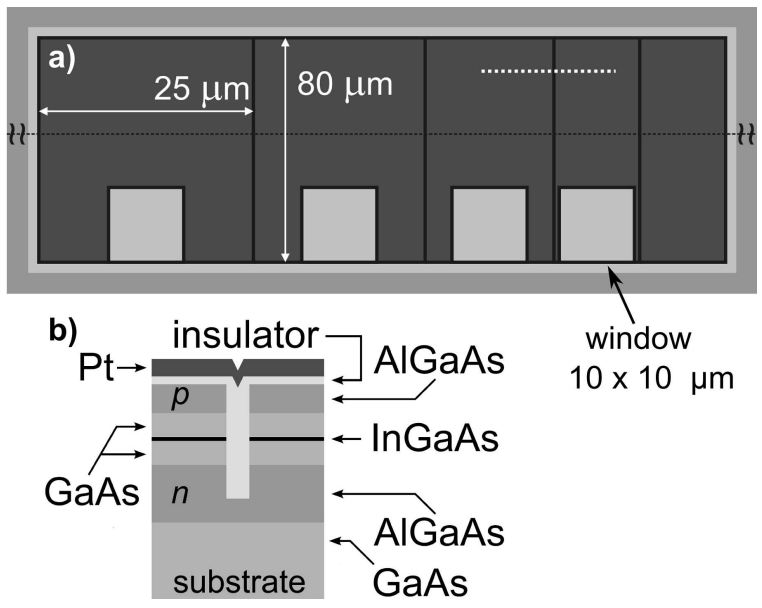

Fig. 2. (a) Planar view of the second test structure (not in scale) with five stripes cut off from the rest of the heterostructure. (b) A cross-section view of the structure in a place marked by a dotted line in part (a). The insulator and platinum were deposited with the use of FIB, with $10 \times 10 \mu \mathrm{m}^{2}$ windows left uncovered by platinum in four stripes.

While the CL detector in scanning electron microscopy (SEM) is sometimes used to measure the EL generated intentionally by external bias in semiconductor structures [5], the present investigation shows that an unexpected (secondary) EL emission during CL measurements may lead to misinterpretation of the detected luminescence. Since the laser beam excitation of similar samples generates the optical/laser beam-induced current (analogous to the EBIC in CL measurements and labeled OBIC or LBIC), the conclusions drawn from the presented CL results are useful for PL technique and for other luminescence measurement techniques as well.

The electron beam exciting a semiconductor structure generates $\mathrm{e}-\mathrm{h}$ pairs, which diffuse and recombine, but if they reach a strong electric field, the electrons and holes are separated. The region of separation acts as an EBIC current source, with the rest of the structure functioning as the load. The beam-induced voltage forward biases the junction and the whole structure. When an electron beam excites the structure in planar view, perpendicularly to the $p-n$ junction, the induced current flows from the current source through lateral resistances $R_{1}$ of layers and vertically through junction resistances $R_{\mathrm{j}}$ [6]. The current spreading depends on the resistance distribution within the structure and increases with the lowering of $R_{\mathrm{l}} / R_{\mathrm{j}}$ ratio. Junction resistances $R_{\mathrm{j}}$ decrease with the increase of induced voltage and current, i.e. with an increase of electron beam current $\left(I_{\text {beam }}\right)$ or energy ( $\left.E_{\text {beam }}\right)$, but lateral resistances $R_{1}$ are independent of them. The flowing current easily generates EL when the excited structure is for example a light emitting diode (LED) or a laser diode. However, if there are structural defects causing nonradiative recombination in the junction, the junction current flows mainly through defected 
sites, which quenches EL. Thus, during the CL measurements under consideration, the spreading of induced EBIC within the semiconductor structure may happen in two ways: (i) to regions where EL emission occurs, (ii) to defected regions, where EL emission is quenched. Moreover, EL increases with the junction voltage and current.

The distribution of induced current within the structure and consequently the spatial distribution of secondary EL depend on distribution of resistances. If the ratio of lateral to junction resistances is low then the induced-current spreading reaches regions at distances much larger than the diffusion length of minority carri- ers. Therefore, the region of light emission may be much wider than that related only to the carrier diffusion and recombination.

Thus, when a structure with a built-in strong electric field is at one point excited by an electron or laser beam, the luminescence may occur not only from this point but from the rest of the structure as well. As a result the information on local properties of the structure may be distorted in two ways: through the impact of resistances all over the structure on the EBIC level (which is competitive with $\mathrm{CL}$ ) and through the EL contribution to the detected luminescence.
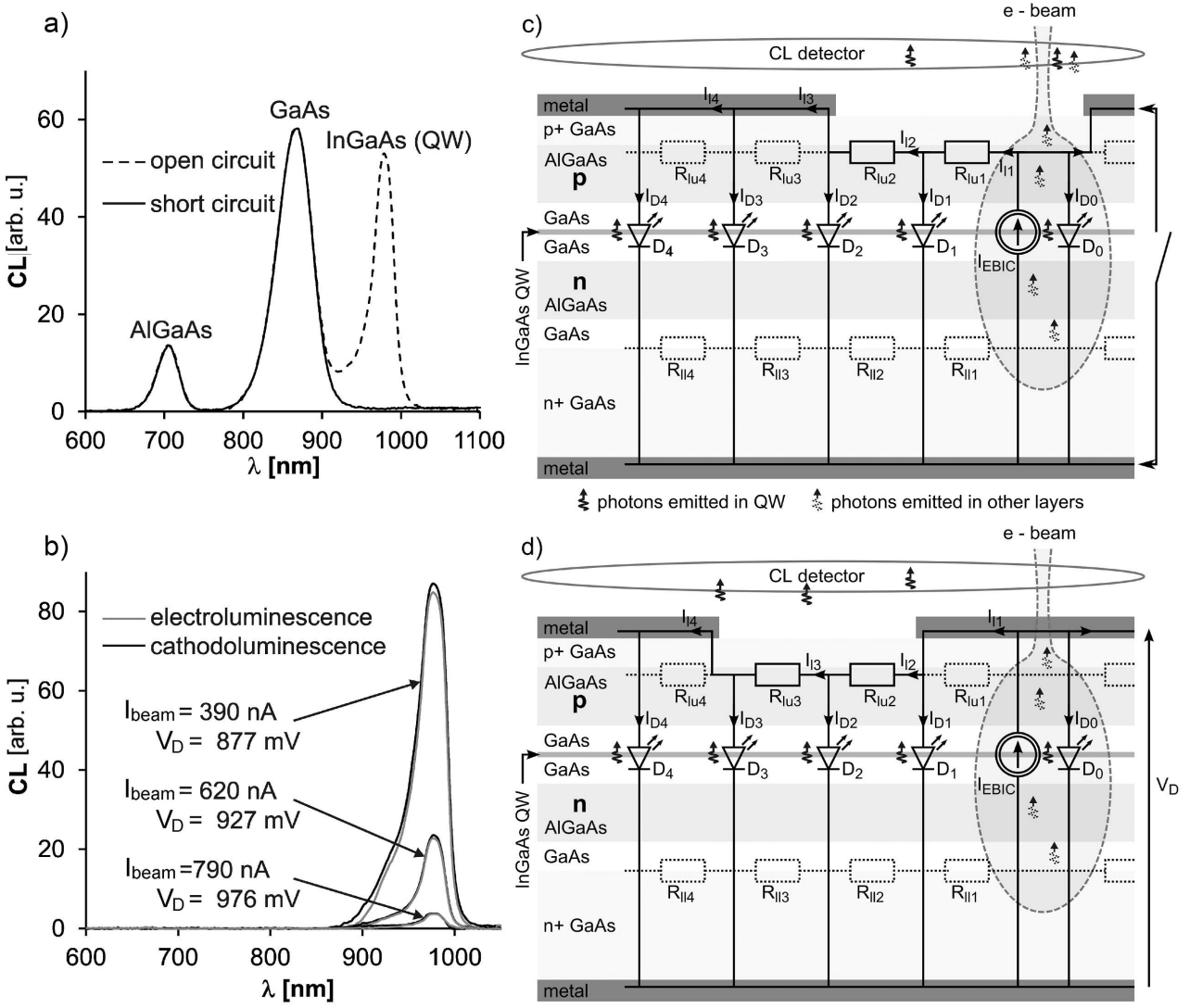

Fig. 3. (a) The CL spectra registered for the first test structure when the electron beam excited the point of mesa without metallization. The cases of either open circuit or short circuit between the external contacts of the structure are shown. (b) Luminescence spectra measured during electron-excitation of the mesa region covered with a thick layer of metallization (seen in Fig. 1a), in configurations of either CL or typical EL measurements (i.e. with the electron beam either on or off, respectively, but in the latter case with the structure externally biased through electrical contacts at the mesa top and the structure bottom). The arrows indicate spectra for three different $I_{\text {beam }}$ current values and corresponding to them $V_{\mathrm{D}}$ voltages measured at the mesa (in CL configuration) or supplied to the mesa (in typical EL configuration): $I_{\text {beam }}=390 \mathrm{nA}$ and $V_{\mathrm{D}}=877 \mathrm{mV} ; I_{\text {beam }}=620 \mathrm{nA}$ and $V_{\mathrm{D}}=927 \mathrm{mV} ; I_{\text {beam }}=790 \mathrm{nA}$ and $V_{\mathrm{D}}=976 \mathrm{mV}$. (c) Simplified electric diagram of the structure with marked local currents for the excitation point placed at mesa without metallization. (d) Simplified electric diagram of the structure with marked local currents for the excitation point placed at mesa covered by metallization.

\section{Results}

The CL spectra detected for configuration of the open circuit between the contacts (for the first test structure), when electrons bombarded the narrow region of mesa un- covered by metallization, show three peaks, at 705,870 and $980 \mathrm{~nm}$, due to emissions from the AlGaAs, GaAs, and InGaAs (i.e. QW) layers, respectively (Fig. 3a). For the short circuit configuration between the contacts, 705 and $870 \mathrm{~nm}$ peaks corresponding to local luminescence 
are still seen, but $980 \mathrm{~nm}$ peak is entirely quenched. The short-circuiting of the structure affects the QW luminescence because of strong electric field produced by the $p-n$ junction, generation of EBIC and its easy outflow in this case. On the contrary, the most of AlGaAs and GaAs layers are outside the strong electric field. Therefore, $\mathrm{e}-\mathrm{h}$ pairs generated by the electron beam in these layers are not separated and their recombination cannot be affected by short-circuiting. Although the electric field separates the $\mathrm{e}-\mathrm{h}$ pairs generated in $\mathrm{QW}$ regardless of the open or short-circuiting of the structure, however the generated EBIC flows differently.

In the open-circuit configuration the EBIC current spreads inside the structure and its flow through the junction (i.e. $I_{\mathrm{D} 0}, I_{\mathrm{D} 1}, I_{\mathrm{D} 2} \ldots$ currents in Fig. $3 \mathrm{~b}$ ) produces detectable luminescence similar to the one produced by the external bias (i.e. to typical EL). Figure 3b shows simplified diagram of the first test structure while it is excited by the electron beam in the region uncovered by the metallization. In the region excited by the electron beam the source of current is generated. The structure consists of several elementary diodes $D_{0}, D_{1}$, etc. (having dynamic resistances $R_{\mathrm{jx}}$ ), lateral resistances of the upper layers $R_{\text {lu1 }}, R_{\text {lu2 }}$, etc. Some of them (marked by dotted lines) are always short-circuited by the upper metallization. The resistances of bottom layers are also marked (by $R_{111}, R_{112}$, etc.), however in our case all of them are always short-circuited by the bottom metallization.

Instead, in the short-circuit configuration (i.e. with attached to contacts an external circuit of negligible resistance) the EBIC outflows to the external circuit so easily that $\mathrm{e}-\mathrm{h}$ pairs do not recombine in InGaAs QW [4], and no EBIC spreading occurs within the structure.

The luminescence spectra measured for the first test structure in either CL or EL configurations (with the electron beam either on or off, respectively, but in the latter case with the structure externally biased through electrical contacts) are shown in Fig. 3a.

When electrons bombarded the metallized part of the mesa, besides CL spectra also the induced voltage $V_{\mathrm{D}}$ was measured between electrical contacts at the mesa top and the structure bottom, using Keithley 617 with input resistance $200 \mathrm{~T} \Omega$. The same voltage was next applied to the contacts for EL measurements. The CL spectra measured at $I_{\text {beam }}$ equal to 390,620 , and $790 \mathrm{nA}$, and the EL spectra at corresponding voltages 877,927 , and $976 \mathrm{mV}$, are shown in Fig. 3b. Only the peak at $980 \mathrm{~nm}$ is seen in both CL and EL spectra. It has been observed previously that peaks of EL spectrum, for heterostructures containing QW, closely correspond to CL spectrum peaks related to the emission from QW [7]. The applied $I_{\text {beam }}$ currents were relatively high in order to enable CL measurements to be taken in places covered with metallization, and also due to the large area of mesas (which leads to a strong EBIC outflow that is competitive with CL emission).

However, if there was only local emission of light, no luminescence signal emitted at places covered by thick metallization (which has a negligible transmittance) could be detected. Instead, the signal detected during CL measurements can be ascribed to the electrical properties of the whole specimen (see Fig. 3d). Regardless narrow regions of mesa uncovered by metallization, EBIC spreading along the mesa was almost undisturbed, because even in places without metallization the current flows along the highly doped upper semiconductor layer. Thus, when the region of CL emission is covered by metallization, a detectable luminescence signal will be due to the sequence of three phenomena: (i) e-h pair separation (in a strong field existing e.g. within InGaAs layer), leading to EBIC generation, (ii) EBIC current spreading over the structure, (iii) EL emission from regions uncovered by metallization.

The second test structure (Fig. 2) enabled to confirm that the described effect is caused by the current outflow and secondary EL phenomenon (and not by the propagation of emitted light along the waveguide layers).

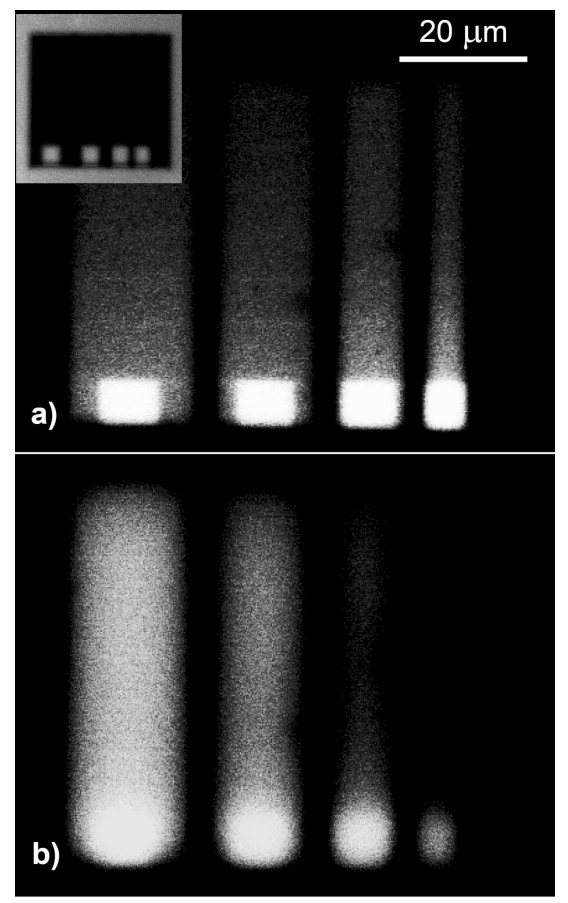

Fig. 4. Monochromatic CL images of the test structure with five stripes prepared by FIB, registered at the wavelength of $980 \mathrm{~nm}$ (emission from InGaAs QW) for beam energy $E_{\text {beam }}=30 \mathrm{keV}$ and for currents $I_{\text {beam }}=35 \mathrm{nA}(\mathrm{a})$ and $225 \mathrm{nA}(\mathrm{b})$. The detector sensitivity at $35 \mathrm{nA}$ was set much higher than at $225 \mathrm{nA}$. The inset shows a smaller CL image of the same structure, registered at the wavelength of $870 \mathrm{~nm}$ (emission from GaAs).

The design of this structure utilized the dependence of current outflow on the resistance distribution in the structure, as opposed to the light propagation. Monochromatic images for $870 \mathrm{~nm}$ (inset in Fig. 4a) and $705 \mathrm{~nm}$ wavelengths (both with a lack of luminescence signal), emitted respectively from GaAs and AlGaAs, show again that the local luminescence from regions cov- 
ered by the Pt layer remains undetected, while the luminescence outside the stripes and at the uncoated windows is the same.

An imaging in SEM technique always correlates the spatial position of the electron beam with the detected (e.g. luminescence) signal, regardless the true source of the signal. The CL images for $980 \mathrm{~nm}$ wavelength (from InGaAs QW) were bright even far from the window, i.e. the luminescence signal was detected also when the beam was placed at regions covered with platinum and far from the window (Fig. 4). The detected luminescence was almost independent of the stripe width at $I_{\text {beam }}=35 \mathrm{nA}$, while it was strongly dependent on the width at $225 \mathrm{nA}$. This confirms the relation of the signal to the electrical properties of the specimen, because the wider stripes have lower lateral resistance $R_{1}$ than the narrower ones, and the impact of different $R_{1}$ becomes more important when the junction resistances decrease, i.e. at higher $I_{\text {beam }}$ values. For the stripe without a window the lack of signal is registered also for $980 \mathrm{~nm}$ wavelength. For stripes with windows the comparison of above images for $980 \mathrm{~nm}$ (Fig. 4a,b) with images for 870 (inset in Fig. 4a) and $705 \mathrm{~nm}$ wavelengths, together with the fact that transmittance of thick $\mathrm{Pt}$ is negligible also for $980 \mathrm{~nm}$ wavelength, confirms that during CL measurements the detected signal of InGaAs QW can be attributed only to the secondary EL emitted through the window in each stripe. On the contrary, the fact that photons emitted from QW may be transmitted along waveguides does not depend on the stripe width or resistance.

Figure 5 shows how the registered luminescence for emission from QW is dependent on the distance between the point of excitation and the window (in a thick overlayer opaque to light). An exemplary case when the e-beam illuminates a point of the stripe far from the window is shown in Fig. 5a. The non-zero intensity of the pixel corresponding to this point (in the middle of the white circle marked as the excitation point in Fig. 5a) results from the spatial distribution of luminescence. This distribution depends on the EBIC spreading and the voltage drop on the lateral resistances $R_{\text {lu }}$. The EBIC current flow through the junction produces secondary EL luminescence all over the structure while only the luminescence emitted in the window region can reach the detector.

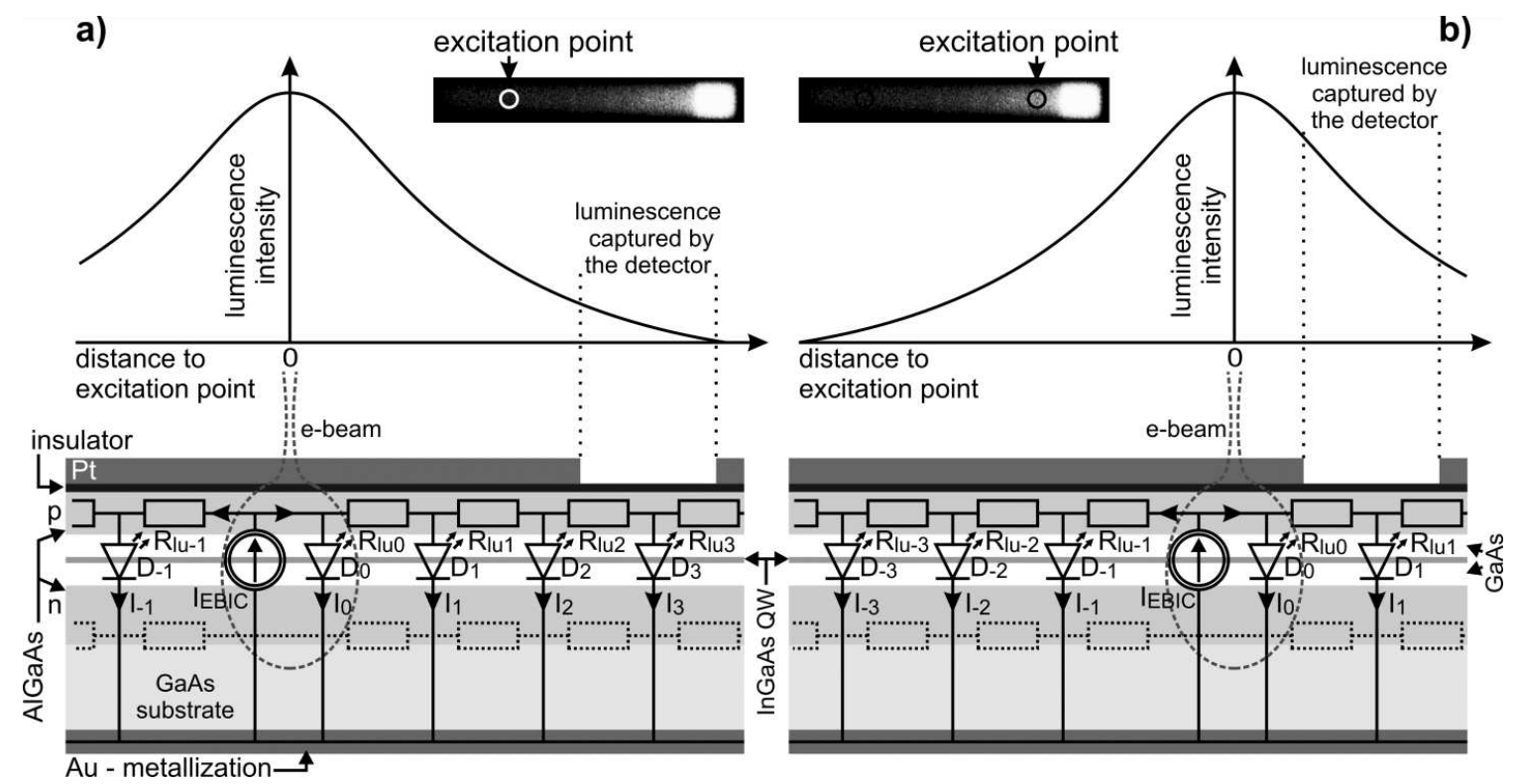

Fig. 5. Interpretation of the results shown in Fig. 4 for two exemplary cases of electron beam excitation $\left(I_{\text {beam }}=35 \mathrm{nA}\right)$ of the second test structure (not in scale) at the $10 \mu \mathrm{m}$ wide stripe with the window (in a thick overlayer opaque to light). (a) The case when the excitation point is far from the window and therefore a small number of photons is captured by the detector. (b) The excitation point is near the window and a large number of emitted photons reach the CL detector. The spatial distribution of luminescence intensity consists of CL signal in the close neighborhood of excitation point (marked "0") and of secondary EL signal (for emission from QW) in other regions. The schematic shape of generation region of electron-hole pairs is drawn at " 0 " distances.

In the simplified diagram shown in Fig. 5a the detected secondary EL is generated by the current $I_{3}$ flowing through the elementary LED $D_{3}$ under the window. In the case shown in Fig. 5b the point of excitation (in the middle of the marked black circle) and region of EBIC generation are placed near to the window. Due to the same spatial distribution of luminescence the intensity of the detected secondary EL (generated by the current $I_{1}$ flowing through the elementary LED $D_{1}$ under the window) is higher than in the case shown in Fig. 5a. Because 
the signals of a SEM detector are correlated with the points of excitations, therefore image pixel corresponding to the point of excitation is brighter in Fig. 5b than in Fig. 5a.

\section{Conclusions}

Strong non-local secondary EL from places distant from the point of electron- or laser-beam excitation may contribute to the total luminescence signal during CL or PL measurements of the semiconductor structures. In addition to the beam power, the contribution also depends on, among other factors, the ratio of lateral and junction resistances $R_{\mathrm{l}} / R_{\mathrm{j}}$ (where $R_{\mathrm{l}}$ has a constant value and $R_{\mathrm{j}}$ changes with the induced voltage), the dependence of induced voltage on the beam power (related to the semiconductor material and structure), the thickness of the overlayers, and the shape, size and condition of the measured structure. When local luminescence is quenched by overlayers and secondary EL is emitted from a window in this cover at location distant from the excitation point, the contribution of secondary EL to the total luminescence is especially large.

To reduce the spreading of the induced current regardless the magnitudes of other parameters (e.g. $E_{\text {beam }}$ and $\left.I_{\text {beam }}\right)$, the cuts in the sample made with FIB technique, such as those used for shaping the stripes (in Fig. 1b), were applied. Our CL measurements showed that such cuts prevent EBIC from spreading outside the limited region (e.g. of stripes), strongly increase the intensity of detected luminescence (even for $I_{\text {beam }}$ of the order of single $\mathrm{nA}$ ) and decrease the contribution of secondary EL.
A useful advice for limiting spreading of induced current during CL (or PL) measurement is also to avoid measuring in regions (e.g. of mesas) with metallization contact or with highly doped layers. Their whole area is in fact short-circuited, which facilitates the spreading of generated EBIC (or LBIC/OBIC) and its easy access to all places (e.g. of current leakage or luminescence generation) in the whole short-circuited area.

\section{Acknowledgments}

The research was partially supported by the Polish National Science Centre grant 2013/09/B/ST7/04218.

\section{References}

[1] A. Gustafsson, M.E. Pistol, L. Montelius, L. Samuelson, J. Appl. Phys. 84, 1715 (1998).

[2] J. Bruckbauer, P.R. Edwards, T. Wang, R.W. Martin, Appl. Phys. Lett. 98, 141908 (2011).

[3] K. Matsuda, T. Saiki, S. Nomura, M. Mihara, Y. Aoyagi, Appl. Phys. Lett. 81, 2291 (2002).

[4] A. Czerwinski, M. Pluska, J. Ratajczak, A. Szerling, J. Kątcki, Appl. Phys. Lett. 93, 041109 (2008).

[5] F. Rossi, G. Salviati, M. Pavesi, M. Manfredi, M. Meneghini, E. Zanoni, U. Zehnder, J. Appl. Phys. 103, 093504 (2008).

[6] M. Pluska, A. Czerwinski, J. Ratajczak, J. Kątcki, L. Marona, R. Czernecki, M. Leszczyński, P. Perlin, J. Microsc. 236, 137 (2009).

[7] A.Y. Nikiforov, G.S. Cargill, S.P. Guo, M.C. Tamargo, J. Appl. Phys. 104, 114506 (2008). 